\title{
Eth2Vec: Learning Contract-Wide Code Representations for Vulnerability Detection on Ethereum Smart Contracts
}

\author{
Nami Ashizawa \\ Naoto Yanai \\ Jason Paul Cruz* \\ n-ashizawa@ist.osaka-u.ac.jp \\ yanai@ist.osaka-u.ac.jp \\ Osaka University \\ Suita, Osaka, Japan
}

\author{
Shingo Okamura \\ okamura@info.nara-k.ac.jp \\ National Institute of Technology, Nara College \\ Yamatokoriyama-shi, Nara, Japan
}

\begin{abstract}
Ethereum smart contracts are programs that run on the Ethereum blockchain, and many smart contract vulnerabilities have been discovered in the past decade. Many security analysis tools have been created to detect such vulnerabilities, but their performance decreases drastically when codes to be analyzed are being rewritten. In this paper, we propose Eth2Vec, a machine-learning-based static analysis tool for vulnerability detection in smart contracts. It is also robust against code rewrites, i.e., it can detect vulnerabilities even in rewritten codes. Existing machine-learning-based static analysis tools for vulnerability detection need features, which analysts create manually, as inputs. In contrast, Eth2Vec automatically learns features of vulnerable Ethereum Virtual Machine (EVM) bytecodes with tacit knowledge through a neural network for natural language processing. Therefore, Eth2Vec can detect vulnerabilities in smart contracts by comparing the code similarity between target EVM bytecodes and the EVM bytecodes it already learned. We conducted experiments with existing open databases, such as Etherscan, and our results show that Eth2Vec outperforms a recent model based on support vector machine in terms of well-known metrics, i.e., precision, recall, and F1-score.
\end{abstract}

\section{CCS CONCEPTS}

- Security and privacy $\rightarrow$ Software security engineering; Software reverse engineering; Vulnerability management.

\section{KEYWORDS}

Ethereum, smart contracts, neural networks, static analysis, code similarity, vulnerability detection

ACM Reference Format:

Nami Ashizawa, Naoto Yanai, Jason Paul Cruz, and Shingo Okamura. 2021. Eth2Vec: Learning Contract-Wide Code Representations for Vulnerability Detection on Ethereum Smart Contracts. In Proceedings of the 3rd ACM International Symposium on Blockchain and Secure Critical Infrastructure

Permission to make digital or hard copies of all or part of this work for personal or classroom use is granted without fee provided that copies are not made or distributed for profit or commercial advantage and that copies bear this notice and the full citation on the first page. Copyrights for components of this work owned by others than the author(s) must be honored. Abstracting with credit is permitted. To copy otherwise, or republish, to post on servers or to redistribute to lists, requires prior specific permission and/or a fee. Request permissions from permissions@acm.org.

BSCI '21, June 7, 2021, Virtual Event, Hong Kong

(c) 2021 Copyright held by the owner/author(s). Publication rights licensed to ACM ACM ISBN 978-1-4503-8400-1/21/06 .. \$15.00

https://doi.org/10.1145/3457337.3457841
(BSCI '21), June 7, 2021, Virtual Event, Hong Kong. ACM, New York, NY, USA, 13 pages. https://doi.org/10.1145/3457337.3457841

\section{INTRODUCTION}

\subsection{Backgrounds}

Ethereum [50] is the largest platform that provides an execution environment for smart contracts, and many distributed applications have been developed and deployed as smart contracts on the Ethereum blockchain. Ethereum smart contracts are programs that are stored on the Ethereum blockchain and are run by the Ethereum Virtual Machine (EVM) as EVM bytecodes ${ }^{1}$.

Given the transparent and decentralized nature of the Ethereum blockchain, the EVM bytecodes of smart contracts deployed on the Ethereum blockchain can be accessed and analyzed by anyone. Unfortunately, this also means that an adversary can abuse smart contracts [52] by analyzing their EVM bytecodes and looking for vulnerabilities. Consequently, attacks on vulnerable smart contracts can occur and possibly cause significant damages, especially when the attacked smart contracts handle assets. For example, the DAO attack is an infamous springboard attack where the attacker/s exploited a vulnerability in the DAO smart contract and stole more than 60 million USD worth of Ether, the cryptocurrency used in Ethereum.

According to literature [52], the security of smart contracts cannot be guaranteed because of the complexity (or lack of complexity) of the programming languages used for creating smart contracts, e.g., Solidity, which is a relatively new language, and the insufficient knowledge of programmers when creating smart contracts. To make matters worse, smart contracts that are successfully deployed on a blockchain cannot be modified, i.e., their source codes cannot be edited or deleted. In the past years, many attacks on deployed smart contracts have been reported [2], and thus making sure that the source code of smart contracts is not vulnerable before they are deployed on a blockchain is desirable. To do this, many analysis tools for vulnerability detection in Ethereum smart contracts have been developed [9].

In this paper, we aim to develop a static analysis tool that can precisely detect various vulnerabilities in smart contract codes with high throughput by analyzing these codes. In static analysis, only the source code of a target to be analyzed is provided as input to identify if the code has vulnerabilities without executing the target

${ }^{1}$ Hereafter, "Ethereum smart contract/s" and "smart contract/s" are used interchangeably but have the same meaning. 
itself. Therefore, ideally, static analysis can be used to prevent vulnerable codes from being deployed.

However, static analysis has two problems in general: (1) accuracy of its vulnerability detection is limited, and (2) its analysis time can be long. For instance, disassembly from EVM bytecodes $[6,35,43,51]$ does not have the capability to identify whether a program is vulnerable or not, and the early literature focuses on simply improving the readability of disassembled codes. In other words, disassembled codes often need to be analyzed manually, consequently increasing the number of false positives and false negatives. Moreover, symbolic execution [8, 27, 46, 49], which extracts control flow graphs (CFGs) from a target code, achieves high accuracy by automating the analysis, but the generation of CFGs needs to search all states such that the target code transits. Therefore, the analysis takes significant amounts of time [48].

A potential solution to the problems described above is machine learning. Static analysis based on machine learning infers whether a given code is vulnerable by learning features of codes. In doing so, the analysis also achieves a versatile analysis within a short time. However, static analysis based on machine learning has two inherent limitations: (1) code rewrites decrease analysis accuracy, and (2) appropriate features of smart contracts are indefinite. In the first limitation, for instance, CFGs with inlined functions are different from those of the original functions. Therefore, identifying the original function with the inlined function as the same codes is challenging. A pair of functions with the same semantics but different structures may generate different analysis results because the differences between structures of the codes strongly affect the analysis. In the second limitation, although features are manually extracted for machine learning, code samples and open knowledge about smart contracts are insufficient. Common knowledge about smart contract features has never been established [52]. Notably, kinds of features representing vulnerabilities in codes of smart contracts are unknown. Besides, features that are robust against differences in code structures are still not obvious. Neural Network is a common approach to extract features implicitly. However, the existing approach based on neural network $[15,31]$ have not solved the above limitations, and are insufficiently robust against code rewrites.

\subsection{Contributions}

In this paper, we propose Eth2Vec, a static analysis tool based on machine learning that identifies smart contract vulnerabilities by learning smart contract code via their EVM bytecode, assembly code, and abstract syntax trees. Eth2Vec has high throughput, high accuracy, and robustness against code rewrites. Eth2Vec is an analysis tool based on a neural network for natural language processing, and it outputs the existence and kind of vulnerabilities in a target smart contract code by only taking the code as input. Using Eth2Vec, a user can analyze code of smart contracts quickly even without expert knowledge on smart contract vulnerabilities.

In terms of analysis of Ethereum smart contracts by using machine learning, a major contribution of this paper is the provision of a method that is robust against code rewrites. Loosely speaking, Eth2Vec overcomes the limitations described in Section 1.1 by leveraging a neural network for natural language processing. Namely,
Eth2Vec can extract features more precisely than a trivial use of a neural network by virtue of the natural language processing that learns the context of each function. Although there are several existing tools $[15,36]$ based on a typical language model for the analysis of Ethereum smart contracts, i.e., Word2Vec [29], Eth2Vec is novel since it achieves robust analysis against code rewrites. To do this, we also developed a module that gives the EVM bytecode and their syntactic information to the neural network as inputs. By virtue of the developed module, Eth2Vec can extract and learn features of code more informatively than the Word2Vec-based tools. Furthermore, we designed a learning methodology based on a neural network for natural language processing which takes the EVM bytecode through the compilation of Solidity code as inputs. (See Section 4 for detail.)

We conducted experiments to evaluate the performance of Eth2Vec. The experimental results show that Eth2Vec can detect vulnerabilities within 1.2 seconds per contract with an average precision of $77.0 \%$. Notably, reentrancy, whose severity is the highest among known vulnerabilities [45], can be detected with $86.6 \%$ precision. Eth2Vec outperforms a typical method [31] in terms of wellknown metrics on vulnerability detection. Moreover, Eth2Vec can detect vulnerabilities of rewritten code, which were not found by the existing Word2Vec-based tool named SmartEmbed [15]. (See Section 5 for detail.) We release the source code of Eth2Vec via GitHub for reproducibility and as reference for future works (https://github.com/fseclab-osaka/eth2vec).

\section{PRELIMINARIES}

In this section, we describe background knowledge to help readers understand our work.

\subsection{Ethereum Smart Contracts}

In Ethereum, there are two kinds of accounts, namely, an externally owned account (EOA) and a contract account. EOAs have a private key that can be used to access the corresponding Ether or contracts. A contract account has smart contract code, which an EOA cannot have, and it does not have a private key. Instead, it is owned and controlled by the logic of its smart contract code. In Ethereum, a smart contract refers to an immutable computer program that is deployed on the blockchain and runs deterministically in the context of the EVM. The immutability property indicates that, similar to any data published on a general blockchain, smart contract codes can be considered as trustworthy, i.e., once deployed, they cannot be changed or deleted. The deterministic property indicates that the execution of the coded functions of smart contracts will produce the same result for anyone who runs them. Once deployed on the blockchain, a contract is self-enforcing and managed by the peers in the network, i.e., its functions are executed when the conditions in the contract are met. A smart contract is given an identity in terms of a contract address. Using this address, it can receive Ether and its functions can be executed. A contract is invoked when its contract address is the destination of a transaction, which is a signed message originating from an EOA, transmitted by the network, and recorded on the blockchain. Such transaction causes a contract to run in the EVM using the transaction (and transaction's data) as input. The data indicate which specific function in the 
contract to run and what parameters to pass to that function. To incentivize peers to execute contract functions, Ethereum relies on gas, which is paid in Ether, to "fuel computations". The amount of gas needed to execute a transaction is relative to the complexity of the computations, thus also preventing infinite loops.

Smart contracts are typically written in a high-level language, such as Solidity [1]. The source code is then compiled to low-level bytecode that runs in the EVM. The EVM is a simple stack-based architecture. Its instruction set is kept minimal to avoid incorrect implementations that could cause consensus problems. The EVM is a global singleton, i.e., it operates like a global, single-instance computer that runs in all peers in the network. Each peer runs a local copy of the EVM to validate the execution of contract functions, and the processed transactions and smart contracts are recorded on the blockchain.

\subsection{Machine Learning}

Machine learning consists of two algorithms, i.e., training and inference. The training algorithm takes data as input to learn their features and optimize parameters inside the model for an objective function. On the other hand, the inference algorithm takes unlearned data as input and infers a similar set of features to that of the training data. When each data is unlabeled, a learning algorithm is called unsupervised learning. The most popular approach to machine learning in the recent years is deep learning, which is based on neural networks and can extract features in a black-box manner. In this paper, we aim to develop a model for learning vulnerable smart contracts to detect vulnerabilities in unlearned smart contracts.

A neural network model is a machine learning model representing connections between neurons, which is a brain mechanism on a human, on a computer. The model approximately expresses complicated non-linear functions on a computer. Two kinds of tasks, or a regression task and a classification task, are treated on a neural network as well-known settings. The regression problem infers continuous numbers from input data, and the classification problem infers a class that an input data belongs to. The feature extraction of Eth2Vec with a neural network for natural language processing is classified as a regression problem. Each input signal of a neural network has a unique weight, and the weight is an element to propagate the importance of the input signal to the output layer. Namely, the impact of input on the output of the neural network becomes large in proportion to the weight of the input. An activation function decides how to activate a sum of input signals of the neural network.

Support Vector Machine (SVM) is a linear binary classifier to classify the input into two classes, and provides significantly high performance for the classification. Furthermore, SVM provides nonlinear classification by combining with the kernel method.

\subsection{Natural Language Processing}

Natural language processing deals with languages that humans use to communicate with each other, and the languages are expressed as texts. Typical applications of natural language processing are, for instance, document classification, machine translation, document summarization, response to questions, and interactive conversation.
In general, "word" is a minimum unit (token) to process a language, and "sentence" is processed together as one unit string. Word is also the smallest unit as components in a sentence from the perspective of grammar or semantics, and each sentence has a cohesive context. A unit string that is shorter than sentence and consists of more than two words is called "phrase", and natural languages sometimes are processed in a unit of phrase. However, splitting a text into word, phrase, and sentence units is not simple and the definition of each unit is non-trivial.

One of the important differences between a text written in a natural language and code written in a programming language is that only the code has syntax and structures. Natural language processing needs to consider the syntax and structure of code for processing the code.

\section{ANALYSIS OF SMART CONTRACTS VIA MACHINE LEARNING}

This section describes the analysis target as the problem setting and its technical difficulty to be tackled in this paper.

\subsection{Analysis Target}

In this paper, we focus on security analysis of the Solidity language as a target of static analysis of Ethereum smart contracts. In particular, we aim to detect the existence of vulnerabilities and classify the kinds of vulnerabilities in the codes to be analyzed. Assume for example that a developer uses a tool that analyzes the smart contracts he/she is developing in local.

Such tool potentially needs to convince the developer that the smart contract being developed does not have any vulnerability even if the developer does not have sufficient knowledge about smart contract vulnerabilities. Therefore, we aim to develop a tool that can detect the existence of vulnerabilities (if there are any) in smart contracts even if only the codes of the smart contracts are given as input. Such a specification for analyzing smart contracts is preferable because standardized knowledge about Ethereum smart contracts is insufficient compared to general programming languages such as $\mathrm{C}$ and Java [52]. Meanwhile, strong obfuscation, i.e., the use of encryption, on Solidity codes is out of the scope of this paper because such an obfuscated code of smart contracts does not exist as far as we know.

Hereafter, we refer to codes written in Solidity as a contract and a file of codes consisting of more than a single contract as a contract file. We also call contracts to evaluate a vulnerability as test contracts and those to learn the vulnerability as training contracts. We call codes obtained from the compilation of the contracts as EVM bytecodes. The largest unit in each contract is a function, and a library function is also identical to a function. Finally, "the blockchain" will be used to refer to the Ethereum blockchain unless otherwise specified. The problem setting in this paper is then formalized as follows:

Problem Formulation: We formalize our approach for analysis of smart contracts as follows. Each contract $c_{i} \in C$ includes vulnerabilities $V_{i}=\left\{v_{1}^{i}, \cdots, v_{l}^{i}\right\} \in \mathcal{V}^{l}$, where $C$ denotes a set of contracts, $\mathcal{V}$ denotes a set of vulnerabilities independent of each other, and $l$ denotes any number. Given any integer $n \in \mathbb{N}$, a combination of a contract and vulnerabilities $C V=\left\{\left(c_{1}, V_{1}\right), \cdots,\left(c_{n}, V_{n}\right)\right\}$ and a 
test contract $c_{t} \in C$ are inputs of a model $M$. Let $\left\{\epsilon_{i}^{c_{t}}\right\}_{i \in[1, d]} \subseteq \mathbb{R}^{d}$ denote the output of the model $M$ which has $d=|\mathcal{V}|$ elements, where $|\mathcal{V}|$ denotes the size of $\mathcal{V}$ and $\epsilon_{i}^{c_{t}}$ denotes a probability about vulnerabilities in $\mathcal{V}$. Our goal is to develop a tool that optimizes $M\left(C V, c_{t}\right) \rightarrow\left\{\epsilon_{i}^{c_{t}}\right\}$.

\subsection{Technical Difficulty}

Although several machine-learning-based static analysis tools for smart contracts have been proposed in literature [25, 26, 31, 42, 48], accuracy of their vulnerability detection is limited even on known vulnerabilities. In other words, accuracy significantly decreases when codes are rewritten from the original codes. Following the problem formulation described above, the limitation described in Section 1.1 as our motivation is formalized as follows: even if $c_{t}$ with a vulnerability $V_{i}$ which is included in $C V$ for any $i \in[1, n]$, $\epsilon_{i}^{c_{t}} \ll \epsilon_{i}^{c_{i}}$ holds for $c_{t} \notin C V$. We call such a situation non-robust.

The limitation related to the accuracy of vulnerability detection described above is caused by the insufficient extraction of features of the existing tools. In general, a machine learning model needs features as inputs, which are manually extracted, and then learns the features explicitly. However, features for representing smart contracts to be analyzed are non-obvious because the history of Ethereum smart contracts is shorter than other general languages such as $\mathrm{C}$ and Java [52]. Intuitively, the limitation about the nonrobustness is denoted as that required of a model $M$ is unknown. Another reason for the insufficient extraction of features is the lack of code samples of smart contracts [52].

\section{DESIGN OF ETH2VEC}

In this section, we present Eth2Vec. We first describe the design concept to overcome the technical difficulty described in the previous section and then present the tool overview and its building blocks. Finally, we present the Eth2Vec model with its objective function.

\subsection{Design Concept}

We aim to solve the technical difficulty by leveraging neural networks for natural language processing. For the natural language processing by machine learning, each word is vectorized as a token, and each sentence is vectorized as a sequence in general. To incorporate natural language processing, we leverage the $P V-D M$ model [22] which vectorizes each paragraph by taking into the context of the paragraph account. More specifically, we extend the PV-DM model whereby code of each contract is vectorized instead of the natural language. Eth2Vec can thus extract features implicitly.

To provide the extended PV-DM model with code of contracts, we also developed a new module named EVM Extractor to obtain syntax and semantics for each contract. For instance, EVM Extractor obtains principal code information, e.g., a function name and start address. Eth2Vec then restricts the amount of the code information to be provided the PV-DM model because the throughput of Eth2Vec may decrease in proportion to the amount of the information. Eth2Vec can thus achieve the feature extraction appropriately and efficiently. Afterwards, Eth2Vec computes the code similarity by utilizing the code information vectorized through neural networks for the natural language processing described above. When the security of code is analyzed, a model learns vulnerable code and it can then detect the vulnerabilities via the similarity of code to be analyzed with the learned code. Therefore, Eth2Vec can analyze vulnerabilities in code even if essential features of the vulnerabilities are unclear.

\subsection{Tool Overview}

Eth2Vec consists of two modules, i.e., PV-DM model [22] for neural networks to deal with paragraphs and EVM Extractor to create inputs of the PV-DM model from Solidity source codes.

The overview of Eth2Vec is shown in Figure 1. First, a PV-DM model is utilized as neural networks to deal with bytecodes. In particular, the PV-DM model executes unsupervised learning by taking JSON files generated from bytecodes as input and then computes the code similarity for each contract. To do this, we developed the EVM Extractor as a module to create inputs of the PV-DM model from EVM bytecodes because the PV-DM model cannot deal with EVM bytecodes initially. More specifically, the EVM Extractor analyzes EVM bytecodes syntactically and creates JSON files for instruction-level, block-level, function-level, and contract-level.

As a result, Eth2Vec takes EVM bytecodes to be analyzed as input, and then returns lists of code clones and their vulnerabilities for contract-level from a user's standpoint. Meanwhile, vulnerabilities in contracts for training data are identified in advance by the use of existing tools [27, 45]. Vulnerabilities for test data are then evaluated by the code similarity with the vulnerable contracts. Eth2Vec outputs vulnerabilities for the function-level in codes to be analyzed. Namely, vulnerable functions can be identified by Eth2Vec.

\subsection{Building Blocks}

Eth2Vec utilizes the PV-DM model [22] and EVM Extractor as building blocks.

4.3.1 PV-DM model. The PV-DM model vectorizes text data for paragraph-level in contrast to Word2Vec [29] to vectorize text data for sentence-level. Intuitively, it outputs vector representations for each word and each paragraph. More concretely, given a text paragraph consisting of multiple sentences, a PV-DM model applies a sliding window over each sentence. The sliding window starts from the beginning of the sentence and moves forward a single word at each step. In doing so, the PV-DM model executes a multiclass inference task such that it maps the current paragraph into a vector and each word in the context into a vector. More precisely, the model averages these vectors and infers the target word from the vocabulary via the softmax function. The architecture of the PV-DM model consists of one hidden layer: for instance, the model obtains vectors for the given input and then compute the average of the vectors and a sigmoid function. The output layer computes a classification result based on a softmax function. Formally, given a text that contains a list of paragraphs $p \in T$, each paragraph $p$ contains a list of sentences $s \in p$, and each sentence is a sequence of $|s|$ words $w_{t} \in s$. Then, the PV-DM model maximizes the log probability as follows:

$$
\sum_{p}^{T} \sum_{s}^{p} \sum_{t=k}^{|s|-k} \log \mathbf{P}\left(w_{t} \mid p, w_{t-k}, \cdots, w_{t+k}\right) .
$$




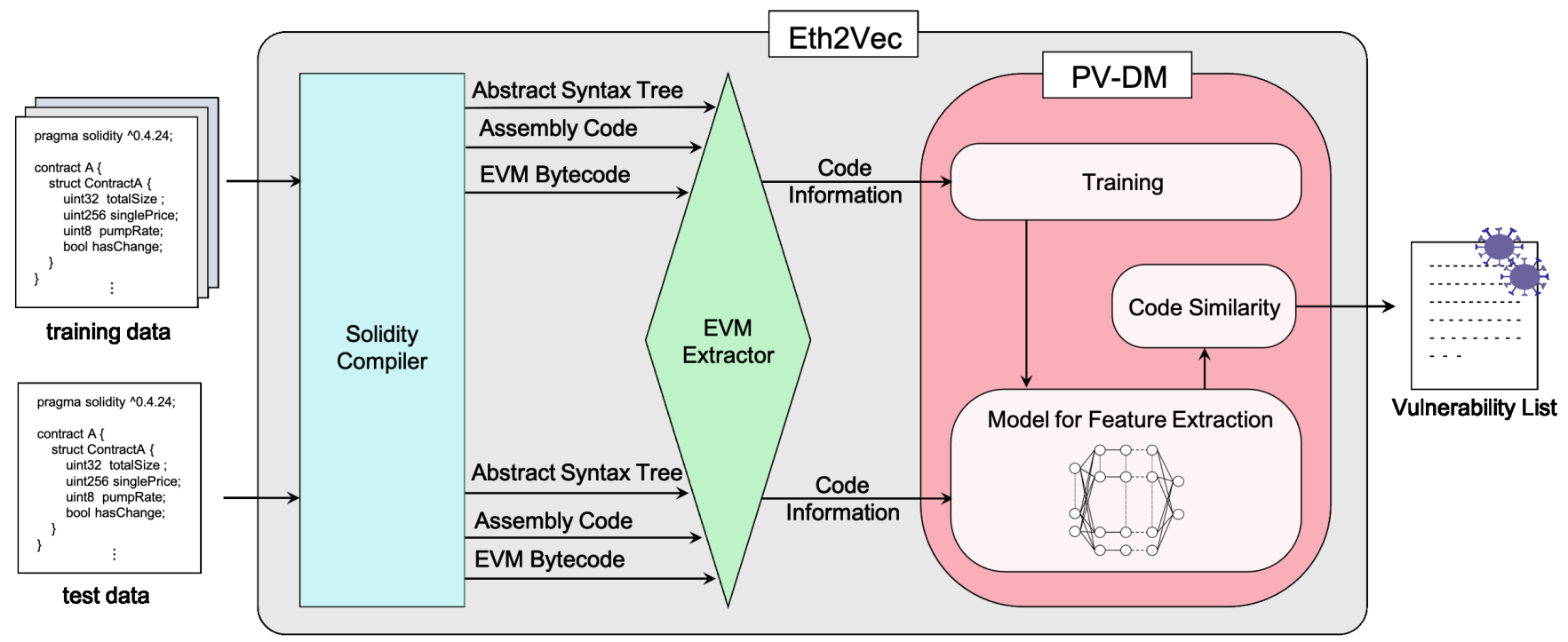

Figure 1: Overview of Eth2Vec

The PV-DM model is initially designed for text data that is sequentially laid out while it can also be used for analysis of assembly code by extending vector representation of language [11].

In this paper, we present a contract-wide representation learning model by extending the PD-DM model in accordance the syntax of EVM bytecode as described in Section 4.4.

4.3.2 EVM Extractor. EVM Extractor is a module that syntactically analyzes EVM bytecodes for PV-DM model. In particular, EVM Extractor parses Solidity files as in the following hierarchical structure:

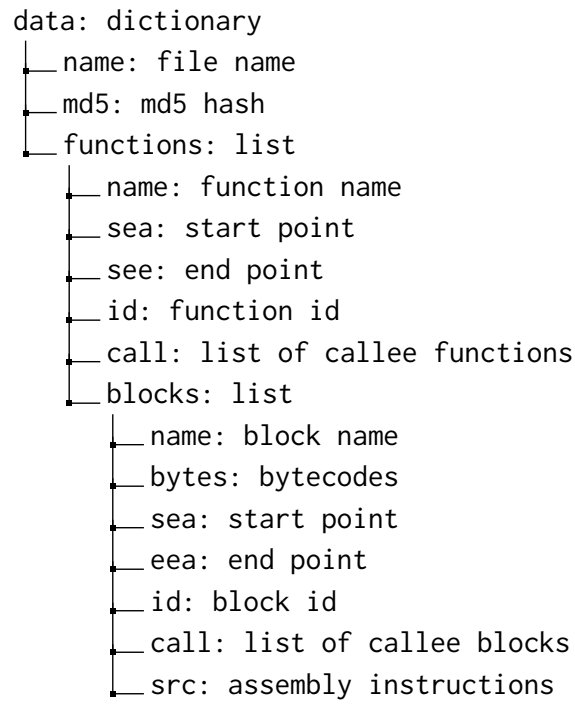

The top-level, data, represents a contract file to be analyzed The second level indicates file-dependent information. The third level indicates function-dependent information. The fourth level indicates a block consisting of multiple instructions. In each level, sea means the start address of the current function and that of the current block. Likewise, see and eea mean the start address of the next function and that of the next block, respectively. In contrast, call represents a callee function (or a callee block) from the current function (or block). Finally, at the bottom level, instructions of EVM bytecodes are stored in src together with their addresses. Meanwhile, a library function is treated as an individual function.

\subsection{Design of Objective Function}

Eth2Vec is a model that learns and infers codes of Ethereum smart contracts through unsupervised learning. Intuitively, it can be regarded as an extension of Word2 Vec with a wider vector representation of codes given its ability to target Ethereum smart contracts.

The objective function of Eth2Vec is designed by extending that of the original PV-DM model described in Equation (1) along with the syntax shown in Section 4.3.2. The following argument is inspired by Asm2Vec [11], which is an extension of the PV-DM model for an assembly language. Given a file of an Ethereum smart contract to be analyzed, Eth2Vec vectorizes the codes for instructionlevel and then computes the code similarity to the learned codes by the training. The process above is executed for block-level related to the instructions, function-level, and contract-level recursively, and therefore Eth2Vec can detect codes with distribution similar to that of the training data. Formally, an objective function is defined as follows:

$$
\sum_{C_{i}}^{\operatorname{Dict}} \sum_{f_{s}}^{\mathcal{U}\left(C_{i}\right)} \sum_{s e q_{i}}^{\mathcal{S}\left(f_{s}\right)} \sum_{i n_{j}}^{\mathcal{I}\left(\operatorname{seq}_{i}\right)} \sum_{t_{c}}^{\mathcal{T}\left(\text { in }_{j}\right)} \log \mathbf{P}\left(t_{c} \mid C_{i}, i n_{j-1}, i n_{j+1}\right),
$$

where we denote by Dict a contract file, by $C_{i}$ a contract, by $f_{s}$ a function, by $s e q_{i}$ a block consisting of multiple instructions, by $i n_{j}$ each instruction, and by $t_{c}$ a token with respect to the current instruction. Likewise, we denote by $\mathcal{U}\left(C_{i}\right)$ multiple functions, by $\mathcal{S}\left(f_{s}\right)$ multiple blocks, by $\mathcal{I}\left(s e q_{i}\right)$ a list of instructions, and by $\mathcal{T}\left(i_{j}\right)$ a list of tokens.

Here, the first summation term, i.e., $\sum_{C_{i}}^{\text {Dict }}$, is included in the second level shown in the previous section, i.e., file name. Equation (2) 
is given contracts and their instructions, and then it maximizes the $\log$ probability for the current token $t_{c}$. Intuitively, the lexical meaning for each contract is computed through the current instruction and its neighbor instructions. Moreover, codes consisting of multiple contracts can also be analyzed by extracting features for each contract.

We describe the objective function to represent the aforementioned intuition in detail below. Given a contract file $\mathrm{Dict}$, a function $f_{s}$ for each contract $C_{i}$ is vectorized and then we denote by $\overrightarrow{\theta_{f_{s}}}$ the vector representation of $f_{s}$. Furthermore, we denote by $C \mathcal{T}$ (in) average of the vector representation of neighbor instructions of in. We then define a concatenation of the vector representation of the instruction itself and that of its operand as follows:

$$
C \mathcal{T}(\text { in })=\overrightarrow{v_{\mathcal{P}(\text { in })}} \| \frac{1}{\mathcal{A}(\text { in })} \sum_{t}^{\mathcal{A}(\text { in })} \overrightarrow{v_{t}},
$$

where $\mathcal{P}($ in $)$ is one operation with respect to in, $\mathcal{A}($ in $)$ is a list of operands with respect to $i n$, and $\|$ is a concatenation of strings. In doing so, for any contract $C_{i}$, by averaging $f_{s}$ and the $j$-th instruction $i n_{j}$ with $C \mathcal{T}\left(i n_{j-1}\right)$ and $C \mathcal{T}\left(i n_{j+1}\right)$, a function $\delta\left(i n_{j}, f_{s}\right)$ to evaluate the joint memory of neighbor instructions in $f_{s}$ is defined as follows:

$$
\delta\left(i n_{j}, f_{s}\right)=\frac{1}{3}\left(\overrightarrow{\theta_{f_{s}}}+C \mathcal{T}\left(i n_{j-1}\right)+C \mathcal{T}\left(i n_{j+1}\right)\right) .
$$

Therefore, the log probability in Equation (2) can be replaced with $\mathbf{P}\left(t_{c} \mid C_{i}, i n_{j-1}, i n_{j+1}\right)=\mathbf{P}\left(t_{c} \mid \delta\left(i n_{j}, f_{s}\right)\right)$. According to the literature [11], by letting $X=\left(\overrightarrow{v_{t_{c}}}\right)^{T} \times \delta\left(i n_{j}, f_{s}\right)$ and utilizing a sigmoid function $\sigma(X)=\frac{1}{1+e^{-X}}$, the above computation can be approximated by the $k$-negative sampling $[22,30]$ as follows:

$$
\begin{gathered}
\sum_{C_{i}} \sum_{f_{s}}^{\text {Dict }} \sum_{\text {seq }_{i}}^{\mathcal{U}\left(C_{i}\right)} \sum_{i n_{j}}^{\mathcal{S}\left(f_{s}\right)} \sum_{t_{c}}^{\mathcal{I}\left(\text { seq }_{i}\right)} \sum^{\mathcal{T}\left(\text { in }_{j}\right)} J(\theta) \\
=\sum_{C_{i}}^{\text {Dict }} \sum_{f_{s}}^{\mathcal{U}\left(C_{i}\right)} \sum_{s e q_{i}}^{\mathcal{S}\left(f_{s}\right)} \sum_{i n_{j}}^{\mathcal{I}\left(\text { seq }_{i}\right)} \sum_{t_{c}}^{\mathcal{T}\left(\text { in }_{j}\right)} \log \mathbf{P}\left(t_{c} \mid \delta\left(i n_{j}, f_{s}\right)\right), \\
J(\theta) \approx \log (\sigma(X))+\sum_{i=1}^{k} \mathbb{E}_{t_{d} \sim P_{n}\left(t_{c}\right)}\left(\llbracket t_{d} \neq t_{c} \rrbracket \log \sigma(X)\right),
\end{gathered}
$$

where $\llbracket t_{d} \neq t_{c} \rrbracket$ is an identity function which returns 1 if the expression inside the function is true or 0 otherwise. On the other hand, $\mathbb{E}_{t_{d} \sim P_{n}\left(t_{c}\right)}$ is a sampling function that samples a token $t_{d}$ in accordance with the noise distribution $P_{n}\left(t_{c}\right)$ from $t_{c}$. We finally utilize Equation (6) as the objective function of Eth2Vec. Intuitively, the function maximizes the probability of a token $t_{c}$ of the current instruction and decreases that of a token $t_{d}$ of the other instructions. Then, we can compute the gradient with respect to $\theta_{f_{s}}$ through the following derivative:

$$
\begin{aligned}
\frac{\partial J(\theta)}{\partial \overrightarrow{\theta_{f_{s}}}} & =\frac{\partial J(\theta)}{\partial X} \frac{\partial X}{\partial \overrightarrow{\theta_{f_{s}}}} \\
& =\frac{\overrightarrow{v_{t_{c}}}}{3}(1-\sigma(X)) \\
& +\frac{\overrightarrow{v_{t_{c}}}}{3} \sum_{i}^{k} \mathbb{E}_{t_{d} \sim P_{n}\left(t_{c}\right)}\left(\llbracket t_{d} \neq t_{c} \rrbracket(1-\sigma(X))\right)
\end{aligned}
$$

Moreover, we can also utilize a more intuitive gradient by approximating the aforementioned gradient computation as follows:

$$
\frac{\partial J(\theta)}{\partial \overrightarrow{\theta_{f_{s}}}} \approx \frac{\overrightarrow{v_{t_{c}}}}{3} \sum_{i}^{k} \mathbb{E}_{t \sim P_{n}\left(t_{c}\right)}\left(\llbracket t=t_{c} \rrbracket-\sigma(X)\right) .
$$

Intuitively, Equation (7) approximates the original derivative described above in a manner that it moves a gradient to the positive if a token is identical to the current instruction or to the negative otherwise. Likewise, the gradient with respect to the vector representation $\vec{v}_{t_{c}}$ of a token on the current instruction can be approximated. Moreover, for the vector representation $\vec{v}_{\mathcal{P}(\text { in })}$ of a token of some operation $\mathcal{P}($ in $)$ and that $\vec{v}_{\mathcal{A}(\text { in })}$ of operands on the current instruction, their gradients can be approximated in a similar manner. These gradients are computed as follows although we omit their derivation:

$$
\begin{aligned}
\frac{\partial J(\theta)}{\partial \overrightarrow{v_{c}}} & \approx\left(\llbracket t=t_{c} \rrbracket-\sigma(X)\right) \cdot \delta\left(i n_{j}, f_{s}\right) \\
\frac{\partial J(\theta)}{\partial \overrightarrow{v \mathcal{P}(j+1)}} & \approx\left(\llbracket t=t_{c} \rrbracket-\sigma(X)\right) \cdot \frac{1}{\mathcal{A}(i n)} \sum_{t}^{\mathcal{A}(i n)} \overrightarrow{v_{t}} \\
\frac{\partial J(\theta)}{\partial \overrightarrow{v_{\mathcal{A}\left(i n_{j+1}\right)}^{\longrightarrow}}} & \approx\left(\llbracket t=t_{c} \rrbracket-\sigma(X)\right) \cdot\left(\overrightarrow{v_{\mathcal{P}}\left(i n_{j+1}\right)}\right)
\end{aligned}
$$

Similar equations hold for the previous instruction $i n_{j-1}$ by replacing $i n_{j+1}$ with $i n_{j-1}$ although we omit the details due to space limitation.

\subsection{Training and Inference}

We now present the training algorithm and the inference algorithm with the gradients described in the previous subsection. First, the training algorithm is shown in Algorithm 1. The goal of the training is to optimize vectors for each instruction belonging to the given contract file as input. Instructions with similar meaning are mapped to a similar position in the vector space through the training. These resulting vectors by the algorithm can be used as features for vector representation of contract-level analysis. The algorithm is an unsupervised training algorithm, and thus the procedure does not require a ground-truth mapping between equivalent contracts.

The aforementioned features can be utilized directly in the inference algorithm shown in Algorithm 2. In particular, for an unseen contract $C_{t} \notin \mathrm{Dict}$, the algorithm first initializes $\overrightarrow{\theta_{f_{t}}}$, which is associated with any function $f_{t}$ belonging to $C_{t}$. Then, the algorithm follows the same procedure as the training algorithm. However, all $\overrightarrow{v_{t}}$, s in the trained model are kept, and $\overrightarrow{\theta_{f_{t}}}$, s are updated following their errors. At the end of the inference, $\overrightarrow{\theta_{f_{t}}}$ is output whereas the vectors for all $C_{i} \in$ Dict remain the same except for $\overrightarrow{\theta_{f_{t}}}$. Finally, to search contracts for a match with the given contract $C_{t}$, the resultant vectors are compared with those of the training contracts using a typical statistical method, e.g., the cosine similarity. 

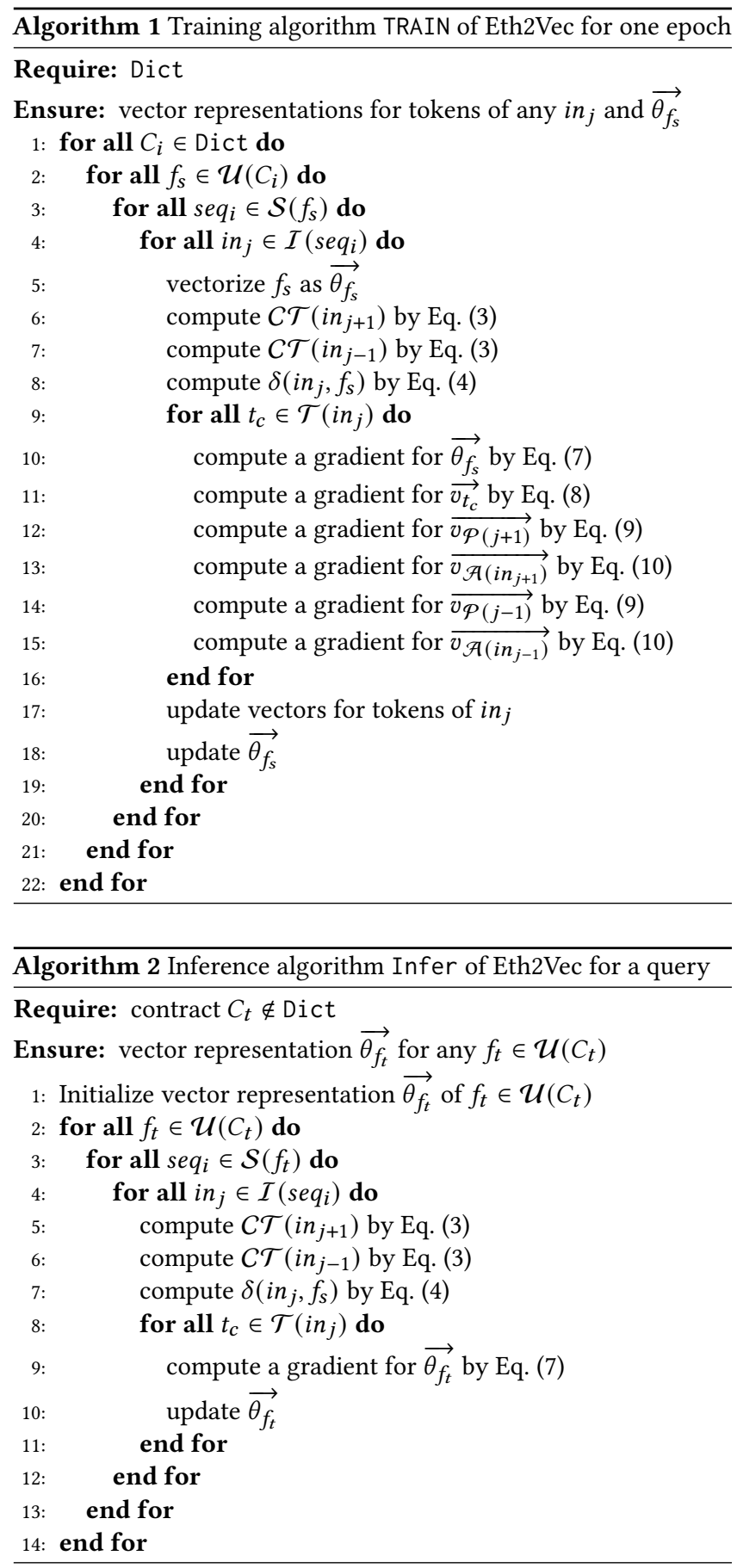

\subsection{Implementation}

We implemented Eth2Vec by utilizing Kam1n $\theta^{2}$ and py-solc $-x^{3}$. First, the main module, PV-DM model, was implemented by Kam1n 0 , which is a server system [10] utilized for binary analysis [11]. We mainly modified the source codes in DisassemblyFactoryIDA.ja

\footnotetext{
${ }^{2}$ Kam1n0 version 2.0.0: https://github.com/McGill-DMaS/Kam1n0-Community

${ }^{3}$ py-solc-x: https://pypi.org/project/py-solc-x/
}

va and ExtractBinaryViaIDA. py. In particular, ExtractBinaryVi aIDA. py initially generates a JSON file extracted from IDA by disassembling binary codes, and then DisassemblyFactoryIDA. java takes the file to store the binary codes within Kam1n 0 . However, IDA cannot use EVM bytecodes for implementing Eth2Vec. Therefore, we changed ExtractBinaryViaIDA. py: For instance, the code information is obtained by compiling a Solidity file with py-solc- $x$ without IDA, and then its resultant assembly codes, abstract syntax tree (AST), and binary codes are extracted.

Meanwhile, the current output display follows the interface of Kam1n0, vulnerabilities are listed as the function-level for each contract. In particular, vulnerabilities of test contracts are displayed with their confidence. We show an output example of Eth2Vec in Appendix A.

We release the source codes of the whole implementation publicly via GitHub (https://github.com/fseclab-osaka/eth2vec).

\section{EXPERIMENTS}

In this section, we describe the experiments we conducted to evaluate Eth2Vec. First, we describe the purpose of the experiments. Then, we discuss the datasets and the training methodologies used for evaluation. Finally, we show the experimental results.

\subsection{Purpose of Experiments}

To evaluate the performance of Eth2Vec, we try to detect vulnerabilities in codes to be analyzed through the training with the known vulnerable contracts. To do this, we first check if Eth2Vec appropriately represents the relationship between codes to be analyzed and codes learned in the training phase. We evaluate clone detection of codes written in the Solidity language to confirm whether Eth2Vec can appropriately extract features of the codes. In doing so, we also evaluate semantic clones based on the lexical-semantic relationship to confirm the robustness of Eth2Vec against code rewrites.

Next, we check whether all the learned vulnerabilities are identified by training the codes and their code similarity with a given code. In doing so, we also evaluate consistency of the vulnerability detection with the results of the clone detection described above. In particular, we check if an output of the vulnerability detection is identical to the vulnerabilities in code identified as clones and if the clones are contained in the clone detection's output.

We confirm that Eth2Vec can extract features precisely and thus can detect vulnerabilities through the experiments mentioned above. We also compare the performance of Eth2Vec with that of the existing work by Momeni et al. [31], which extracts features manually, as a baseline.

\subsection{Experimental Setting}

As mentioned above, experiments are conducted in two stages, i.e., clone detection and vulnerability detection of smart contracts. We describe the setting for each experiment below. We first describe the dataset and the baseline in detail.

5.2.1 Dataset. We collect 5,000 contract files from Etherscan ${ }^{4}$, which is an open database of smart contracts, as a dataset utilized in the experiments. These 5,000 files are also identical to files

\footnotetext{
${ }^{4}$ https://etherscan.io/
} 
utilized in a recent work [15]. We then utilize only the files that can be compiled by solidity version -0.4 .11 as a compiler. The dataset contains 95,152 contracts and 1,193,868 blocks.

5.2.2 Baseline. We compare the performance of Eth2Vec with that of the scheme based on support vector machine (SVM) by Momeni et al. [31] as a baseline. Although there are several versatile results based on machine learning $[15,26,48]$, which can detect various vulnerabilities, their source codes are unpublished or we were unable to build their source codes in our environment. We thus adopt the SVM-based method by Momeni et al. which we were able to reproduce from scratch.

Momeni et al. extracted 16 features from an abstract syntax tree (AST) of an Ethereum smart contract. We utilize 15 of these features excluding hexadecimal addresses because hexadecimal addresses cannot be obtained from source codes, i.e., without deployment. Other features are described in Appendix B.

5.2.3 Clone Detection. We check if Eth2Vec can precisely detect clones of test contracts as input by training contracts. In particular, in the 10-fold cross-validation, 500 test contracts are randomly chosen, and the remaining 4,500 contracts are utilized as the training contracts. This process is iterated 10 times. Meanwhile, a threshold of the code similarity to detect clones is 0.8 .

In the setting mentioned above, precision is utilized as an evaluation metric. For clones for each function, i.e., function-level clones, which are output by a threshold more than 0.8 , we check if a function in a contract, which has the highest similarity, is indeed a clone of the given input. To compare the performance of Eth2Vec, we also evaluate the SVM-based method described above by manually labeling each function. Meanwhile, clones in this experiment correspond to the type-I to type-IV clones in literature [38], and semantic clones are identical to the type-IV clones.

5.2.4 Vulnerability Detection. We check if Eth2Vec can precisely detect vulnerabilities in test contracts as input by learning the training contracts. In particular, we check whether vulnerabilities output by Eth2Vec with a threshold 0.8 about the test contracts are identical to the true vulnerabilities in the contracts.

In this experiment, we adopt the 10 -fold cross-validation similar to that in the clone detection experiment. We also confirm the vulnerabilities in the test contracts as ground truth of the experiment by utilizing Oyente [27] and SmartCheck [45]. In this experiment, we adopt well-known metrics, i.e., precision, recall, and F1-score.

\subsection{Results of Clone Detection}

The results of the clone detection by Eth2Vec are shown in Table 1 . According to the table, Eth2Vec can implicitly extract more features than the SVM-based method [31]. For instance, the values of the standard deviation by Eth2Vec are significantly smaller than those by the SVM-based method. Intuitively, this means that feature extraction by Eth2Vec is more stable, and therefore Eth2Vec is able to represent features on various codes. When the clone detection performance is compared to EClone [26] as a reference, the detection rate of EClone with a threshold of 0.4 was $58.2 \%$. Although we did not implement EClone by ourselves as described in Section 5.2.2,
Table 1: Precision of Clone Detection of Eth2Vec. This table shows the average and standard deviation of 10 executions of precision measurement on 10 -fold cross-validation. The "SVM w/o few clones" column shows the results of the SVMbased setting that were computed by removing test datasets with few clones. The numbers are truncated to one decimal place.

\begin{tabular}{|l|c|c|c|}
\hline & Eth2Vec & SVM [31] & $\begin{array}{c}\text { SVM w/o } \\
\text { few clones }\end{array}$ \\
\hline \hline Average & $74.9 \%$ & $34.6 \%$ & $42.7 \%$ \\
\hline Standard Deviation & 0.9 & 34.6 & 43.6 \\
\hline
\end{tabular}

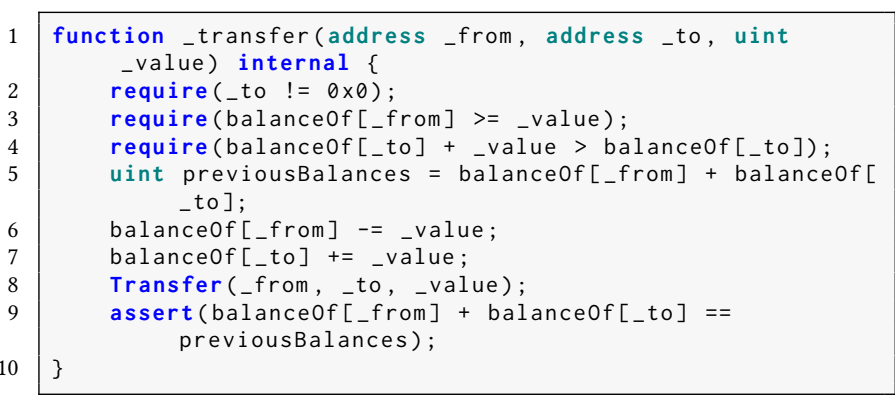

\section{Listing 1: Original function of Transfer}

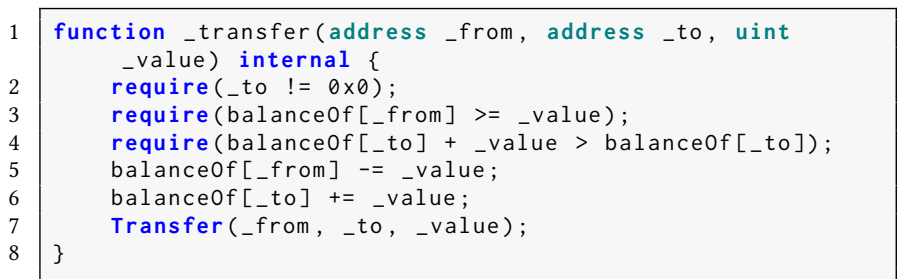

Listing 2: Clone of the original function of Transfer found by Eth2Vec

we believe that Eth2Vec can detect clones of Ethereum smart contracts better than EClone because of its more sophisticated feature extraction.

When we checked an output of Eth2Vec in detail, it contained semantic clones. We show a concrete example in Listing 1 and Listing 2. The output of Eth2Vec, i.e., Listing 2, is a clone of the original function shown in Listing 1 excluding lines 5 and 9. Listing 2 is identical to a type-III clone of Listing 1 according to the definitions in [38]. This means that Eth2Vec can detect clones precisely, even those of rewritten codes.

The standard deviation of SVM is especially larger than that of Eth2Vec because the difference between the accuracy of detecting each clone is overlarge. Concretely, SVM detects a clone with 91.3\% precision. On the other hand, SVM detects another clone with $0 \%$ precision. In particular, the ratio of clones detected with $0 \%$ precision is high, and then the average of SVM is lower than the standard deviation of SVM. Meanwhile, the precision of the SVMbased method is small because the test datasets contain few clones in several executions. The SVM-based method infers negatives for 


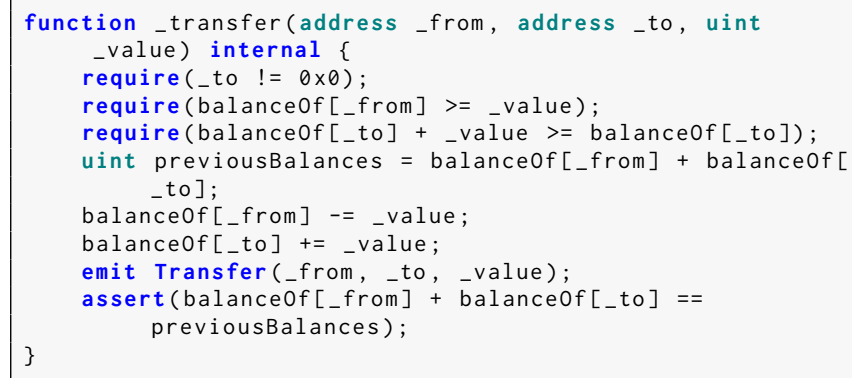

Listing 3: Vulnerability example by Eth2Vec to the original function

the given test data under such situation. Therefore, the precision becomes 0 , resulting in a low average and a more extensive standard deviation. Nonetheless, when we re-compute the precision by removing the results with the few clones among ground-truth, the precision is updated to $42.7 \%$.

\subsection{Results of Vulnerability Detection}

The results of the vulnerability detection by Eth2Vec are shown in Table 2.

First, the standard deviations of Eth2Vec are lower than those of the SVM-based method. This means that Eth2Vec diminishes the effect of difference among features of vulnerabilities because Eth2Vec can detect the vulnerabilities more stably than the SVMbased method. Therefore, Eth2Vec can precisely extract features and thus, it can take into account various vulnerabilities.

Second, we confirm that Eth2Vec is also robust against the dispersion of data. In particular, the appearance of vulnerabilities with high severity, i.e., reentrancy and time dependency, is low in the current dataset. Consequently, the number of the corresponding labels is less, and the recall of the SVM-based method became low. By contrast, the recall of Eth2Vec is more stable than that of the SVM-based method even against vulnerabilities, whose frequency of appearance is low, in comparison with the other vulnerabilities.

Moreover, when we checked an output of Eth2Vec in detail, we found a concrete example, as shown in Listing 3 . The code is output as a clone of Listing 1 and the code similarity of Listing 1 with Listing 3 is lower than that with Listing 2. As difference between these codes, Listing 1 and Listing 2 do not have vulnerabilities while Listing 3 contains the integer overflow vulnerability. Specifically, line 4 in Listing 3 is different from Listing 1 with respect to an expression statement, and the statement in Listing 3 is identical to a typical form of integer overflow ${ }^{5}$.

The aforementioned example indicates that the vulnerability detection of Eth2Vec is robust against code rewrites. Although Listing 1 and Listing 3 seem to be more similar than Listing 1 and Listing 2, Listing 3 is different from a "precise" clone of Listing 1 because Listing 3 contains the integer vulnerability, which is not included in Listing 1. Therefore, Eth2Vec returns a higher code similarity for Listing 2 and Listing 1 than for Listing 3 and Listing 1 . We thus confirm that Eth2Vec has robust vulnerability detection.

\footnotetext{
${ }^{5}$ https://github.com/ConsenSys/mythril/wiki/Integer-Overflow
}

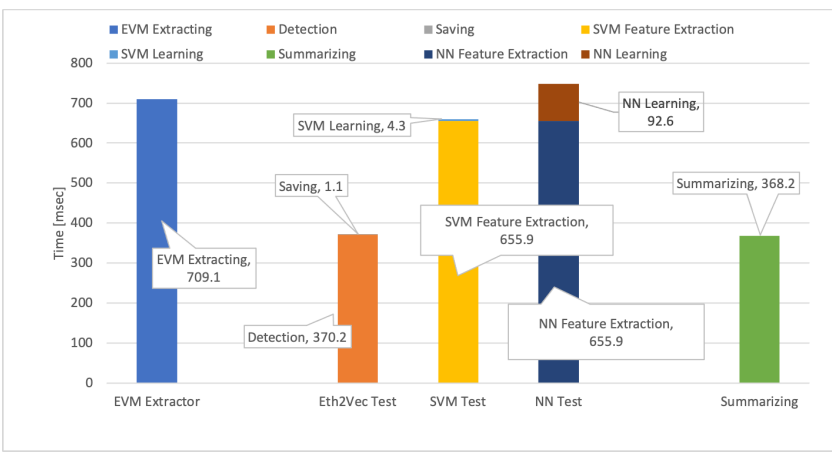

Figure 2: Throughput of Eth2Vec: We measured the throughput of Eth2Vec for each process. The "Eth2Vec Test" is identical to the vulnerability detection, which contains the detection itself and saving detection results. The "Summarizing" represents the processing time for the display process of the saved data. The entire process until a user obtains an output is a summation of EVM Extractor, Eth2Vec Test, and Summarizing. In this measurement, we randomly chose 20 contract files, where a threshold is 0.8 and five clones, i.e., candidates of vulnerabilities, at maximum for each function are output.

\subsection{Inference Throughput}

The throughput of the inference by Eth2Vec is shown in Figure 2. The processing time for vulnerability detection depends on the number of functions and that for displaying the detection results, i.e., summarizing by Kam1 $\mathrm{n} \theta$, depends on the number of detected clones. According to the current measurement, the detection throughput of Eth2Vec is faster than that of the SVM-based method except for the process of EVM Extractor. Eth2Vec can analyze within about 0.371 seconds per contract in comparison with about 0.660 seconds per contract by the SVM-based method. Meanwhile, the processing time for displaying the detection results is longer than the detection itself. A long analysis needed 5.6 seconds for detection, 0.015 seconds for saving, and 3.5 seconds for summarizing, where the number of contracts is 9 and the number of functions is 94 .

We also measured the throughput of existing symbolic execution tools, Oyente [27] and SmartCheck [45], which are publicly available and provides reproducibility. The results are shown in Table 3. According to the table, the Eth2Vec can detect vulnerabilities faster than Oyente and SmartCheck. More specifically, the standard deviation of Oyente is significantly large, and therefore the analysis time becomes exponentially large when a file containing a large number of contracts is analyzed. On the other hand, SmartCheck can detect vulnerabilities faster than Oyente since it considers only a subset of all execution paths. Nevertheless, Eth2Vec is still faster than SmartCheck. Moreover, the F1-score of SmartCheck calculated in accordance with the values in the paper [45] is $39.1 \%$ and lower than that of Eth2Vec.

\section{DISCUSSION}

In this section, we discuss further extensions and limitations of Eth2Vec. 
Table 2: Vulnerability Detection Results of Eth2Vec: In the following table, we measured well-known metrics for both Eth2Vec and the SVM-based method [31]. The values of the averages and standard deviations are computed from the values of each row. The "Severity" column refers to literature [45]. The numbers are truncated to one decimal place.

\begin{tabular}{|c|c||c|c|c||c|c|c|}
\hline \multirow{2}{*}{ Vulnerability } & \multirow{2}{*}{ Severity } & \multicolumn{3}{c||}{ Eth2Vec } & \multicolumn{3}{c|}{ SVM [31] } \\
\cline { 3 - 9 } & Precision [\%] & Recall [\%] & F1-score [\%] & Precision [\%] & Recall [\%] & F1-score [\%] \\
\hline \hline Reentrancy & 3 & 86.6 & 54.8 & 61.5 & 30.0 & 7.8 & 12.3 \\
\hline Time Dependency & 2 & 75.2 & 17.0 & 27.3 & 55.0 & 2.8 & 5.3 \\
\hline ERC-20 Transfer & 1 & 95.6 & 58.4 & 72.4 & 89.0 & 95.3 & 92.0 \\
\hline Gas Consumption & 1 & 48.0 & 29.0 & 32.4 & 10.0 & 3.1 & 4.7 \\
\hline Implicit Visibility & 1 & 68.9 & 82.0 & 74.8 & 71.5 & 77.5 & 73.8 \\
\hline Integer Overflow & 1 & 89.9 & 57.6 & 70.1 & 84.9 & 73.1 & 78.3 \\
\hline Integer Underflow & 1 & 74.6 & 56.0 & 63.7 & 75.1 & 39.2 & 50.0 \\
\hline \hline \multicolumn{2}{|c||}{ Average } & 77.0 & 50.7 & 57.5 & 59.3 & 42.7 & 45.2 \\
\hline \multicolumn{2}{|c||}{ Standard Deviation } & 14.7 & 19.7 & 18.0 & 27.3 & 36.4 & 34.7 \\
\hline
\end{tabular}

Table 3: Comparison of Throughput with Existing Tools: We measured the throughput of Eth2Vec and those of several analysis tools. Oyente [27] and SmartCheck [45] are symbolic execution tools. Each value represents the average time per contract. The numbers are truncated to two decimal place.

\begin{tabular}{|l|c|c|c|c|}
\hline & Eth2Vec & $\begin{array}{c}\text { SVM } \\
{[31]}\end{array}$ & $\begin{array}{c}\text { Oyente } \\
{[27]}\end{array}$ & $\begin{array}{c}\text { SmartCheck } \\
{[45]}\end{array}$ \\
\hline \hline Average [sec] & 0.37 & 0.66 & 3.52 & 0.50 \\
\hline Standard Deviation & 0.59 & 0.60 & 11.45 & 0.71 \\
\hline
\end{tabular}

\subsection{Further Extensions}

Possible extensions of Eth2Vec are described below.

First, important features for detecting vulnerabilities can be clarified by introducing the interpretability of neural networks. Although the current specification of Eth2Vec does not provide insight into the important features, the importance for each feature can be evaluated on Eth2Vec by utilizing a universal method, e.g., permutation importance [5].

Second, the output of Eth2Vec with respect to vulnerabilities can be extended from the current function-level to the instruction-level. Asm2Vec [11], which is an application of Kam1n0, outputs vulnerabilities for the instruction-level by utilizing Abas tractCloneSearch Handler of Kam1n0. Eth2Vec will be able to output vulnerabilities for the instruction-level by incorporating the module.

\subsection{Limitations}

Several limitations of Eth2Vec, which will be improved in future works, are described below. First, the current construction is in the unsupervised setting, and the supervised setting is not implemented. According to Hill et al. [20], the supervised setting seems to be more suitable for the classification of natural language processing than the unsupervised setting. Intuitively, evaluating a kind of vulnerability contained in codes is a classification problem about vulnerabilities. Therefore, the performance of Eth2Vec can be improved by utilizing the supervised setting.
Second, the current work only detected vulnerabilities in contracts included in the training dataset utilized in our experiment. There are potentially many vulnerable contracts that have already been deployed, and thus it would be ideal if all deployed contracts are analyzed to determine potential vulnerabilities.

Finally, the current construction does not support inter-contract analysis where multiple contracts are interconnected. For instance, Rodler et al. [37] presented a new kind of vulnerability by utilizing CALL and CREATE instructions, which requires inter-contract analysis $[8,49]$. Thus, the attacks by Rodler et al. are out of the scope of Eth2Vec currently. Our future work will aim to extend the current construction of Eth2Vec and overcome the limitations described above.

\section{RELATED WORKS}

In this section, we describe related works in terms of security analysis by machine learning.

\subsection{Security Analysis of Ethereum by Machine Learning}

As machine learning-based analysis, ContractWard [48] and the tool by Momeni et al. [31] are based on support vector machine (SVM) and random forest, whose features are extracted manually by an analyst. Hence, identifiable vulnerabilities are limited due to insufficient feature extraction as described in Section 3. As research on automated feature extraction, VulDeeSmartContract [36] has been proposed by combining Word2Vec with long-short term memory (LSTM), but it specializes only in reentrancy vulnerability. Eth2Vec can be considered as a tool dealing with more versatile vulnerabilities in a similar approach.

Next, EClone [25] is a tool that detects code clones of Ethereum smart contracts through the computation of code similarity. Although EClone does not utilize neural networks, a symbolic analysis tool suitable for vector computations of codes has also been proposed. The detectability of Eth2Vec can be improved potentially by combining it with EClone. 
In the neural network-based approach, there is ILF [18] which automatically generates an input of fuzzing test via neural networks. Loosely speaking, ILF learns codes by extracting features via symbolic execution. Since ILF learns outputs by symbolic execution, it is an entirely different tool from Eth2Vec, which learns codes themselves.

Finally, SmartEmbed [15] is a tool that identifies bugs of smart contract codes by leveraging FastText, which represents the codes as vectors. SmartEmbed is the closest work to Eth2Vec. However, SmartEmbed did not discuss an objective function and training algorithm explicitly and just vectorizes codes in accordance with Word2Vec and FastText. Consequently, there are several code samples which are unidentified as clones, as shown in the paper $[15]^{6}$. In contrast, Eth2Vec can precisely extract features of codes with a vast vocabulary and thus can detect vulnerabilities even after codes are rewritten. Nevertheless, SmartEmbed is an elegant work that also discussed code repairing as well as versatile bugs. Interested readers are advised to read the SmartEmbed paper.

\subsection{Other Analysis Tools for Ethereum}

To the best of our knowledge, symbolic execution [27] is the principal approach for analysis of Ethereum smart contracts. Since symbolic execution deals with unknown variables as symbolic variables, it is potentially suitable for analysis of smart contracts, which utilizes information outside codes [8], i.e., blockchain. Hence, many tools have been proposed so far [7, 13, 24, 32-34, 46]. The primary motivation of recent works aims to extend analysis areas, e.g., inter-contract analysis and contract creation. Although symbolic execution consumes a longer time in comparison with a machine learning-based approach as described in Section 1.1, the vulnerability detection of Eth2Vec may be improved by combining it with such tools.

Another approach for smart contract analysis is formal verification [4] which deduces whether a program satisfies a specification via predicate logic. In general, formal verification can provide precise analysis by representing the security in a mathematical way. However, the verification itself is a rigid and challenging work. Many works just formalized a specification of Ethereum and did not achieve the analysis of vulnerabilities [16, 17, 17, 19, 21, 47]. As the latest work, Grishchenko et al. presented a formal verification tool named eThor [39] that can analyze vulnerabilities in a versatile way.

Finally, there are several tools on dynamic analysis which execute codes themselves [14, 37]. However, using dynamic analysis, an analyst needs to implement and execute attack patterns by him/herself to prevent vulnerabilities in advance. Although a universal attack pattern that can capture various attacks was proposed [14], an analyst still needs to have knowledge about attacks on smart contracts. Therefore, analysts with the ability of utilizing a dynamic analysis are limited, and thus static analysis is more reasonable.

\footnotetext{
${ }^{6}$ Although the authors of [15] describe that the samples are not clones, these sample are type-IV clones following the definition in [38].
}

\subsection{Security Applications of Natural Language Processing}

We briefly describe natural language processing applications to the binary analysis and cybersecurity defense as further related works. After Shin et al. [41] proposed binary analysis based on neural networks, many works for binary analysis based on natural language processing, such as Word2Vec, have been proposed in recent years $[11,12,28,53]$. These works are based on neural networks. The closest work to Eth2Vec is Asm2Vec [11], which focuses on assembly language. Eth2Vec can be seen as an extension of the implementation of Asm2Vec. The source code of Asm2Vec is publicly available and readers are advised to read that paper in conjunction with our work. We also note that the same construction can be implemented with other tools described above.

Likewise, natural language processing is another attractive area in cybersecurity. Walk2Friends, which performs a social relation inference attack [3], and DarkEmbed [44], which learns low dimensional distributed representations of darkweb/deepweb discussions, are examples of natural language processing. Other examples are Log2Vec [23] and Attack2Vec [40], which learn more generalized cybersecurity information. These tools apply natural language processing to cybersecurity areas, while Eth2 Vec is an application to Ethereum smart contracts.

\section{CONCLUSION}

In this paper, we proposed Eth2Vec, a static analysis tool based on machine learning that detects vulnerabilities in Ethereum smart contracts. The most striking property of Eth2Vec is the automated feature extraction for each contract by leveraging neural networks for natural language processing. Consequently, by extracting features implicitly and incorporating lexical semantics between contracts, the vulnerabilities can be detected with $77.0 \%$ precision even after the codes are rewritten. Moreover, reentrancy, which is one of the most important vulnerabilities, can be detected with $86.6 \%$ precision. We also demonstrated that Eth2Vec outperforms the SVM-based method by Momeni et al. [31] in terms of precision, recall, and F1-score. We are releasing the implementation of Eth2Vec via GitHub as well (https://github.com/fseclab-osaka/eth2vec).

\section{ACKNOWLEDGMENTS}

This research was supported in part by the Japan Society for the Promotion of Science KAKENHI Numbers 18K18049, and Innovation Platform for Society 5.0 at MEXT.

\section{REFERENCES}

[1] [n.d.]. Security Considerations Solidity 0.5 .11 documentation. https://solidity.readthedocs.io/en/v0.5.11/security-considerations.html.

[2] Nicola Atzei, Massimo Bartoletti, and Tiziana Cimoli. 2017. A survey of attacks on ethereum smart contracts (sok). In Proc. of POST 2017 (LNCS, Vol. 10204). Springer, 164-186.

[3] Michael Backes, Mathias Humbert, Jun Pang, and Yang Zhang. 2017. Walk2friends: Inferring Social Links from Mobility Profiles. In Proc. of CCS 2017. ACM, 1943-1957.

[4] Karthikeyan Bhargavan, Antoine Delignat-Lavaud, Cédric Fournet, Anitha Gollamudi, Georges Gonthier, Nadim Kobeissi, Natalia Kulatova, Aseem Rastogi, Thomas Sibut-Pinote, Nikhil Swamy, et al. 2016. Formal verification of smart contracts: Short paper. In Proc. of PLAS 2016. ACM, 91-96.

[5] Leo Breiman. 2001. Random Forests. (2001), 5-32. 
[6] Lexi Brent, Anton Jurisevic, Michael Kong, Eric Liu, Francois Gauthier, Vincent Gramoli, Ralph Holz, and Bernhard Scholz. 2018. Vandal: A scalable security analysis framework for smart contracts. arXiv preprint arXiv:1809.03981 (2018).

[7] Ting Chen, Xiaoqi Li, Xiapu Luo, and Xiaosong Zhang. 2017. Under-optimized smart contracts devour your money. In Proc. of SANER 2017. IEEE, 442-446.

[8] Yuchiro Chinen, Naoto Yanai, Jason Paul Cruz, and Shingo Okamura. 2020. RA Hunting for Re-Entrancy Attacks in Ethereum Smart Contracts via Static Analysis. In Proc. of Blockchain 2020. IEEE, 327-336.

[9] Monika Di Angelo and Gernot Salzer. 2019. A survey of tools for analyzing ethereum smart contracts. In Proc. of DAPPCON 2019. IEEE, 69-78.

[10] Steven H.H. Ding, Benjamin C.M. Fung, and Philippe Charland. 2016. Kam1n0: MapReduce-Based Assembly Clone Search for Reverse Engineering. In Proc. of KDD 2016. ACM, 461-470.

[11] Steven HH Ding, Benjamin CM Fung, and Philippe Charland. 2019. Asm2Vec: Boosting Static Representation Robustness for Binary Clone Search against Code Obfuscation and Compiler Optimization. In Proc. of IEEE S\&P 2019. IEEE, 472-489.

[12] Yue Duan, Xuezixiang Li, Jinghan Wang, and Heng Yin. 2020. DEEPBINDIFF: Learning Program-Wide Code Representations for Binary Diffing. In Proc. of NDSS 2020. Internet Society.

[13] Josselin Feist, Gustavo Grieco, and Alex Groce. 2019. Slither: A Static Analysis Framework for Smart Contracts. In Proc. of WETSEB 2019. IEEE, 8-15.

[14] Christof Ferreira Torres, Mathis Steichen, Robert Norvill, Beltran Fiz Pontiveros, and Hugo Jonker. 2020. ÆEGIS: Shielding Vulnerable Smart Contracts Against Attacks. In Proc. of AsiaCCS 2020. ACM, 584-597.

[15] Zhipeng Gao, Lingxiao Jiang, Xin Xia, David Lo, and John Grundy. 2020. Checking Smart Contracts with Structural Code Embedding. IEEE Transactions on Software Engineering (2020), 1-1 (Early Access).

[16] Ilya Grishchenko, Matteo Maffei, and Clara Schneidewind. 2018. Foundations and Tools for the Static Analysis of Ethereum Smart Contracts. In Proc. of CAV 2018 (LNCS, Vol. 10981). Springer, 51-78.

[17] Ilya Grishchenko, Matteo Maffei, and Clara Schneidewind. 2018. A semantic framework for the security analysis of ethereum smart contracts. In Proc. of POST 2018 (LNCS, Vol. 10804). Springer, 243-269.

[18] Jingxuan He, Mislav Balunoviundefined, Nodar Ambroladze, Petar Tsankov, and Martin Vechev. 2019. Learning to Fuzz from Symbolic Execution with Application to Smart Contracts. In Proc. of CCS 2019. ACM, 531-548.

[19] Everett Hildenbrandt, Manasvi Saxena, Nishant Rodrigues, Xiaoran Zhu, Philip Daian, Dwight Guth, Brandon Moore, Daejun Park, Yi Zhang, Andrei Stefanescu, et al. 2018. KEVM: A complete formal semantics of the ethereum virtual machine. In Proc. of CSF 2018. IEEE, 204-217.

[20] Felix Hill, Kyunghyun Cho, and Anna Korhonen. 2016. Learning Distributed Representations of Sentences from Unlabelled Data. In Proc. of NAACL HLT 2016. ACL, 1367-1377.

[21] Sukrit Kalra, Seep Goel, Mohan Dhawan, and Subodh Sharma. 2018. ZEUS Analyzing Safety of Smart Contracts.. In Proc. of NDSS 2018. Internet Society.

[22] Quoc Le and Tomas Mikolov. 2014. Distributed representations of sentences and documents. In Proc. of ICML 2014. 1188-1196.

[23] Fucheng Liu, Yu Wen, Dongxue Zhang, Xihe Jiang, Xinyu Xing, and Dan Meng. 2019. Log2vec: A Heterogeneous Graph Embedding Based Approach for Detecting Cyber Threats within Enterprise. In Proc. of CCS 2019. ACM.

[24] Han Liu, Chao Liu, Wenqi Zhao, Yu Jiang, and Jiaguang Sun. 2018. S-gram towards semantic-aware security auditing for ethereum smart contracts. In Proc. of ASE 2018. ACM, 814-819.

[25] Han Liu, Zhiqiang Yang, Yu Jiang, Wenqi Zhao, and Jiaguang Sun. 2019. Enabling Clone Detection For Ethereum Via Smart Contract Birthmarks. In Proc. of ICPC 2019. IEEE, 105-115.

[26] Han Liu, Zhiqiang Yang, Chao Liu, Yu Jiang, Wenqi Zhao, and Jiaguang Sun. 2018. EClone: Detect Semantic Clones in Ethereum via Symbolic Transaction Sketch. In Proc. of ESEC/FSE 2018. ACM, 900-903.

[27] Loi Luu, Duc-Hiep Chu, Hrishi Olickel, Prateek Saxena, and Aquinas Hobor. 2016. Making smart contracts smarter. In Proc. of CCS 2016. ACM, 254-269.

[28] Luca Massarelli, Giuseppe Antonio Di Luna, Fabio Petroni, Roberto Baldoni, and Leonardo Querzoni. 2019. SAFE: Self-Attentive Function Embeddings for Binary Similarity. In Proc. of DIMVA 2019 (LNCS, Vol. 11543), Roberto Perdisci, Clémentine Maurice, Giorgio Giacinto, and Magnus Almgren (Eds.). Springer, 309-329.

[29] Tomás Mikolov, Kai Chen, Greg Corrado, and Jeffrey Dean. 2013. Efficient Estimation of Word Representations in Vector Space. In Proc. of ICLR 2013.

[30] Tomas Mikolov, Ilya Sutskever, Kai Chen, Greg S Corrado, and Jeff Dean. 2013. Distributed representations of words and phrases and their compositionality. In Proc. of NIPS 2013. 3111-3119.

[31] Pouyan Momeni, Yu Wang, and Reza Samavi. 2019. Machine Learning Model for Smart Contracts Security Analysis. In Proc. of PST 2019. IEEE, 1-6.

[32] Mark Mossberg, Felipe Manzano, Eric Hennenfent, Alex Groce, Gustavo Grieco, Josselin Feist, Trent Brunson, and Artem Dinaburg. 2019. Manticore: A UserFriendly Symbolic Execution Framework for Binaries and Smart Contracts. arXiv preprint arXiv:1907.03890 (2019).

[33] B Mueller. 2018. Smashing smart contracts. In Proc. of HITBSECCONF 2018.
[34] Ivica Nikolić, Aashish Kolluri, Ilya Sergey, Prateek Saxena, and Aquinas Hobor. 2018. Finding the greedy, prodigal, and suicidal contracts at scale. In Proc. of ACSAC 2018. ACM, 653-663.

[35] Robert Norvill, Beltran Borja Fiz Pontiveros, Radu State, and Andrea Cullen. 2018. Visual emulation for Ethereum's virtual machine. In Proc. of NOMS 2018. IEEE, $1-4$.

[36] Peng Qian, Zhenguang Liu, Qinming He, Roger Zimmermann, and Xun Wang. 2020. Towards Automated Reentrancy Detection for Smart Contracts Based on Sequential Models. IEEE Access 8 (2020), 19685-19695.

[37] Michael Rodler, Wenting Li, Ghassan O Karame, and Lucas Davi. 2019. Sereum: Protecting Existing Smart Contracts Against Re-Entrancy Attacks. In Proc. of NDSS 2019. Internet Society.

[38] Chanchal Kumar Roy and James R. Cordy. 2007. A Survey on Software Clone Detection Research. School of Computing TR 2007-541, Queen's University 541, 115 (2007), 64-68.

[39] Clara Schneidewind, Ilya Grishchenko, Markus Scherer, and Matteo Maffei. 2020. EThor: Practical and Provably Sound Static Analysis of Ethereum Smart Contracts. In Proc. of CCS 2020. ACM, 621-640.

[40] Yun Shen and Gianluca Stringhini. 2019. ATTACK2VEC: Leveraging Temporal Word Embeddings to Understand the Evolution of Cyberattacks. In Proc. of USENIX Security 2019. USENIX Association, 905-921.

[41] Eui Chul Richard Shin, Dawn Song, and Reza Moazzezi. 2015. Recognizing Functions in Binaries with Neural Networks. In Proc. of USENIX Security 2015. USENIX Association, 611-626.

[42] Jingjing Song, Haiwu He, Zhuo Lv, Chunhua Su, Guangquan Xu, and Wei Wang. 2019. An Efficient Vulnerability Detection Model for Ethereum Smart Contracts. In Proc. of NSS 2019 (LNCS, Vol. 11928). Springer, 433-442.

[43] Matt Suiche. 2017. Porosity: A decompiler for blockchain-based smart contracts bytecode. In Proc. of DEFCON 2017, Vol. 25. 11.

[44] Nazgol Tavabi, Palash Goyal, Mohammed Almukaynizi, Paulo Shakarian, and Kristina Lerman. 2018. Darkembed: Exploit prediction with neural language models. In Proc. of AAAI 2018. AAAI Press, 7849-7854.

[45] Sergei Tikhomirov, Ekaterina Voskresenskaya, Ivan Ivanitskiy, Ramil Takhaviev, Evgeny Marchenko, and Yaroslav Alexandrov. 2018. Smartcheck: Static analysis of ethereum smart contracts. In Proc. of WETSEB 2018. ACM, 9-16.

[46] Christof Ferreira Torres, Julian Schütte, et al. 2018. Osiris: Hunting for integer bugs in ethereum smart contracts. In Proc. of ACSAC 2018. ACM, 664-676.

[47] Petar Tsankov, Andrei Dan, Dana Drachsler-Cohen, Arthur Gervais, Florian Buenzli, and Martin Vechev. 2018. Securify: Practical security analysis of smart contracts. In Proc. of CCS 2018. ACM, 67-82.

[48] Wei Wang, Jingjing Song, Guangquan Xu, Yidong Li, Hao Wang, and Chunhua Su. 2020. ContractWard: Automated Vulnerability Detection Models for Ethereum Smart Contracts. IEEE Transactions on Network Science and Engineering (2020), 1-1 (Early Access).

[49] Konrad Weiss and Julian Schütte. 2019. Annotary: A Concolic Execution System for Developing Secure Smart Contracts. In Proc. of ESORICS 2019 (LNCS, Vol. 11735). Springer, 747-766.

[50] Gavin Wood. [n.d.]. Ethereum: A secure decentralised generalised transaction ledger Byzantium Version. https://ethereum.github.io/yellowpaper/paper.pdf.

[51] Yi Zhou, Deepak Kumar, Surya Bakshi, Joshua Mason, Andrew Miller, and Michael Bailey. 2018. Erays: reverse engineering ethereum's opaque smart contracts. In Proc. of USENIX Security 2018. Usenix Association, 1371-1385.

[52] Weiqin Zou, David Lo, Pavneet Singh Kochhar, Xuan-Bach D Le, Xin Xia, Yang Feng, Zhenyu Chen, and Baowen Xu. 2019. Smart Contract Development: Challenges and Opportunities. IEEE Transactions on Software Engineering (2019), 1-1 (Early Access).

[53] Fei Zuo, Xiaopeng Li, Patrick Young, Lannan Luo, Qiang Zeng, and Zhexin Zhang. 2019. Neural Machine Translation Inspired Binary Code Similarity Comparison beyond Function Pairs. In Proc. of NDSS 2019. Internet Society.

\section{A OUTPUT EXAMPLE BY ETH2VEC}

We show an example of an output by Eth2Vec in Fig. 3. The interface of Eth2Vec follows that of Kam1n0 [10] as described in Section 4.6.

For a test contract given as input, only vulnerable functions in the contract are displayed as a list of functions. Each function is displayed as a function name and a contract name, and then the detail of the detection result is shown by choosing the function. Then, a list of vulnerabilities is displayed as the function-level result. The top-level represents the test contract's name, and the second level represents a function chosen by a user in the test contract. The third level shows a list of functions in the training contracts, whose similarity is high with respect to the function on the second 
level, in descending order. Meanwhile, a user can decide the number of functions detected by Eth2Vec when a test contract is given as input to Eth2Vec. As a result, a training contract is shown in the manner of the third level, i.e., a function name, a contract name, a name of contract file, and vulnerabilities in the contract as long as the contract has the vulnerabilities. The user confirms if each contract or function in a test contract as input is vulnerable from the output of Eth2Vec.

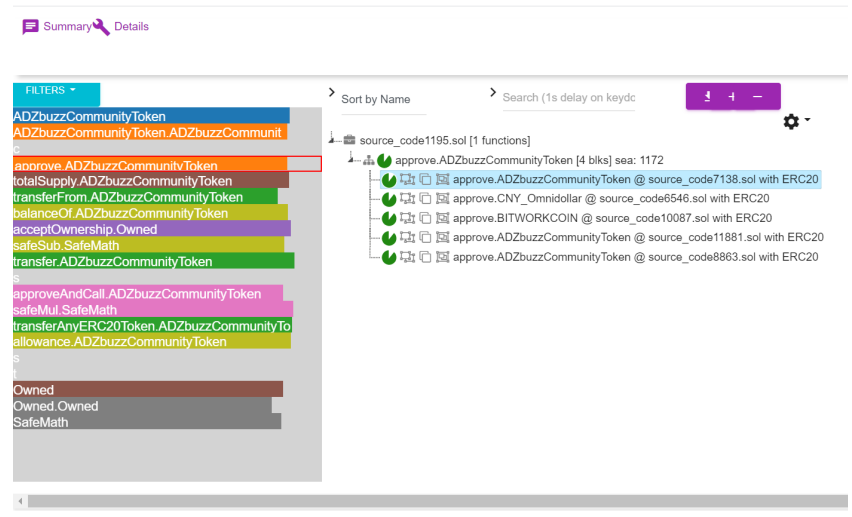

Figure 3: Output example by Eth2Vec: The left-side on the windows displays a list of functions contained in each contract and the right-side shows a list of vulnerabilities included in the chosen function.

\section{B FEATURE SELECTION BY MOMENI ET AL.}

Momeni et al. [31] extracted 16 features from an abstract syntax tree (AST) of an Ethereum smart contract. Among them, we did not adopt Hexadecimal addresses as described in Section 5. The whole list of vulnerabilities is as follows, where all the information can be obtained from AST except for hexadecimal addresses: lines of codes; contract definitions; function definitions: binary operations; function calls; blocks; expression statements; event definitions; bytes; elementary type addresses; modifier definitions; placeholder statements; modifier invocation; approve function definitions; constant values; and hexadecimal addresses. 